\title{
The Effects of 6 Minutes Walking Exercise on the Secondary Mitral Regurgitation
}

\section{Ramazan Asoğlu1, Adem Atıcı2, Hasan Ali Barman3, Emin Asoğlu4, Melike Zehra Buğra5}

\footnotetext{
1Adıyaman University Training and Research Hospital, Clinic of Cardiology, Adiyaman, Turkey

${ }^{2}$ Taksim First Aid Hospital, Clinic of Cardiology, İstanbul, Turkey

3̇̇stanbul Okmeydanı Training and Research Hospital, Clinic of Cardiology, İstanbul, Turkey

4Mardin State Hospital, Clinic of Cardiology, Mardin, Turkey

5ंstanbul University İstanbul Faculty of Medicine, Department of Cardiology, İstanbul, Turkey
}

\section{Abstract}

Background: Mitral Regurgitation (MR) is usually insidious due to progressive remodeling of atrial and left ventricle (LV). In secondary MR, the mitral valve apparatus are structurally normal but dysfunctional because of ischemic or non-ischemic cardiomyopathy. The aim of this study was to identify echocardiographic MR variables associated with 6 Minute Walk (6MW) exercise in patients with non-ischemic cardiomyopathy.

Methods: This prospective study involved 81 consecutive patients with clinically stable non-ischemic LV dysfunction (ejection fraction lower than 40\%) and at least mild MR who were able to perform symptom limited 6MW exercise. All the patients underwent standard Doppler echocardiography examination and each patient had a recent normal coronary angiography. All patients were evaluated for their functional capacities, stratified by New York Heart Association classification and underwent echocardiography evaluation before $6 \mathrm{MW}$ aerobic exercise and after 6 months. The severity of regurgitation established by a comprehensive evaluation of color and conventional Doppler criteria.

Results: Proximal isovelocity surface area (PISA) decreased significantly from $42(17-64)$ to $29(12-47) \mathrm{mm} 2(\mathrm{p}<0.001)$. Regurgitant orifice area calculated by the PISA method decreased significantly from $27(16-41)$ to $20(10-34) \mathrm{mm} 2(\mathrm{p}<0.001)$. The regurgitant volume calculated by the quantitative Doppler method, and vena contracta width decreased significantly from $0.43(0.20$ $0.60)$ to $0.40(0.20-0.50) \mathrm{cm}(\mathrm{p}<0.001)$. There was no significance difference at baseline and after six months for LV end diastolic diameter and diastolic function parameters.

Conclusion: We conclude that a simple, easily reproducible $6 \mathrm{MW}$ exercise might supplement the assessment of the grade of MR in secondary MR patients.

Keywords: 6-minute walk exercise, mitral regurgitation, heart failure, non-ischemic cardiomyopathy

Asoğlu R., Atıcı A., Barman HA., Asoğlu E., Buğra MZ. The Effects of 6 Minutes Walking Exercise on the Secondary Mitral Regurgitation. 


\section{Introduction}

Mitral regurgitation (MR) is the second most prevalent valvulopathy in Europe after aortic stenosis (1). MR is insidious due to progressive remodeling of the atrial and left ventricle (LV). Many patients with systolic dysfunction have secondary MR which influences the ventricular function in patients with systolic heart failure (2). With secondary MR, the mitral valve apparatus is normal but dysfunctional because of ischemic or nonischemic cardiomyopathy. It is well known that exercise capacity is impaired in secondary MR, even when only mild (3). Exercise may change the presence of MR and 6-minute walk test (6MW) which is simple, cheap, reliable, repeatable, and can be performed by almost all the patients (4). We investigated our hypothesis whether 6MW exercise-induced changes in secondary MR.

We aimed to identify echocardiographic MR variables associated with $6 \mathrm{MW}$ in patients with non-ischemic cardiomyopathy. We used quantitative methods to measure MR changes after $6 \mathrm{MW}$ aerobic exercise, three times a week for six months and investigated whether exercise influences the degree of MR in these patients.

\section{Materials and Methods}

Eighty-one patients were diagnosed clinically stable, non-ischemic LV dysfunction (ejection fraction (EF) lower than 40\%) and at least mild MR at İstanbul Faculty of Medicine cardiologic department between January 2016 and June 2017 and were enrolled consecutively. These patients could perform symptom-limited 6MW exercise three times a week for six months. The 12-lead electrocardiogram (ECGs) were obtained $(0.5$ to $150 \mathrm{~Hz}$, $25 \mathrm{~mm} / \mathrm{sec}, 10 \mathrm{~mm} / \mathrm{mV}$ ) and each patient had a recent normal coronary angiography. All the patients underwent a standard Doppler echocardiography examination by Vivid 7 ultrasound system [(General Electric-Vingmed, Milwaukee, WI, United States of America (USA)]. The severity of regurgitation was established by a comprehensive evaluation of color and conventional Doppler criteria. Exclusion criteria were functional
MR (ischemic etiology), myocarditis, atrial fibrillation and poor acoustic window, inability to perform walk test, hypertrophic cardiomyopathy, renal impairment, significant respiratory disease, prior valvular surgery and concomitant moderate or severe valve disease. All patients were evaluated for their functional capacities, stratified by New York Heart Association (NYHA) classification and underwent echocardiography evaluation before $6 \mathrm{MW}$ aerobic exercise and six months after again. Pharmacologic therapy included an angiotensin-converting enzyme inhibitor in all 75, a diuretic in 69, a beta-blocker in 78 and spironolactone in 57 patients. The study was carried out in accordance with the Declaration of Helsinki. We obtained written informed consent from the patients. The ethics committee approved the study.

\section{Echocardiographic Measurements}

Echocardiography was performed, and images were obtained with a $2.5-\mathrm{MHz}$ transducer in the parasternal and apical views. All the patients underwent twodimensional (2D), M-mode and color Doppler transthoracic echocardiography using standard views. 2D parasternal long/short-axis and apical views were performed for mitral anterior/posterior leaflets assessment, and a colored Doppler was used to assess the presence and the degree of severity of the MR. Two diameters of the mitral valve were used for the quantitative Doppler method; one measured from the parasternal long axis and the other from the apical four-chambers view. Both diameters were measured at the base of the leaflets, one frame after which begin to close after their initial opening (5). The MR quantification was performed according to the current recommendations, using both semi-quantitative and quantitative parameters (6).

$2 \mathrm{D}$ echocawrdiography was performed in the apical four-chamber view by using harmonic tissue imaging to optimize endocardial definition. The area-length method was used to calculate end-diastolic and end-systolic LV volumes and the EF (7). The frame captured at the R-wave of the ECG was selected as the end-diastolic frame, and the frame with the smallest LV cavity was considered as the end-systolic frame. 
MR was quantified by two methods: quantitative Doppler echocardiography and proximal isovelocity surface area (PISA) (8). The results from both methods were averaged to calculate effective regurgitant orifice (ERO) and regurgitation volume (RV). Color flow imaging of MR was optimized with a small color angle from the apical window. The image of the mitral valve was expanded using the regional expansion selection. The color flow zero baselines were shifted downward to increase hemispheric PISA. The negative aliasing velocity usually 20 to $40 \mathrm{~cm} / \mathrm{s}$ - was adjusted to obtain satisfactory hemispheric PISA. The MR severity was measured by the PISA method and the most optimal PISA half circle was obtained from the apical four-chamber view in all the patients. The proximal isovelocity radius was measured from at least three frames with the optimal flow convergence. The PISA radius was measured in the mid systole (9). The largest radius, usually in mid-systole, was selected for analysis.

The ERO area was calculated by the standard formula (5). The value of the ERO was obtained by dividing the RV with the MR inflow velocity time integral (VTI) area measured with continuous wave (CW) Doppler at the fourchamber view. During the measurements of ERO and RV with the PISA method in the patients with secondary MR, changes in regurgitation orifice throughout systole were considered (10). To avoid overestimation of the ERO, we measured the radius of the PISA half circle during systole that corresponded to the maximal regurgitation velocity on the CW recording of the VTI of the regurgitant jet.

Doppler vena contracta (VC) width was measured from the apical four-chamber view (11) in each patient. The largest VC diameter was measured. The frame rate in color imaging was maximized by selecting the narrowest sector angle and regional expansion selection was used.

The VTI area at the mitral valve area level was measured by pulse wave (PW) Doppler during the diastole. We took great care in placing the sample volume at the level of the annulus in diastole. For the VTI measurement, the brightest signal of the PW tracing was used (8). The diastolic inflow volume through the mitral valve was calculated by multiplying the mitral valve area with the VTI area during diastole.

Left ventricular (LV) EF was measured by using the biplane Simpson method (12). In the longitudinal parasternal view of the left atrium (LA) diastolic diameter was measured. The mitral $\mathrm{E}$ and $\mathrm{A}$-wave velocities were measured with pulsed-wave Doppler. The ratio of early (E) to the late (A) trans-mitral velocities (E/A) and deceleration time of $\mathrm{E}$ velocity were obtained using pulsed wave Doppler in the apical four-chamber view. The E velocity was measured at the septal mitral annulus in the apical four-chamber view.

All the measurements were performed with the view sector maximally zoomed over the respective valve, and mitral valve diameters were measured. The mean from at least three consecutive beats ( 5 in atrial fibrillation) was taken for each measurement. We took care in placing the sample volume correctly at the level of the annulus in diastole.

\section{Statistical Analysis}

Statistical analysis was performed using a statistical software program (SPSS for Windows, version 20.0; SPSS Inc, Chicago, Illinois, USA). Categorical variables were presented as frequencies with percentages, and continuous variables are presented as mean $\pm \mathrm{SD}$, median and inter-quartile ranges. Differences between before and after 6MW exercise were evaluated with Paired t-test or the Wilcoxon test for parameters with a normal or nonnormal distribution, respectively, as determined with the Kolmogorov Smirnov test. The Pearson correlation test was used to analyze the association between variables. A probability value of $\mathrm{p}<0.05$ was considered significant.

\section{Results}

The baseline characteristics of the 81 patients with non-ischemic MR are presented below (Table 1). The MR group consisted of 81 consecutive patients $63 \%(n=51)$ men, mean age $45.85 \pm 9.86$ years, mean follow up $9.16 \pm 2.5$ years with chronic non-ischemic pathology. The patients 
had 44\% ( $\mathrm{n}=36)$ Hypertension (HT), 14\% $(\mathrm{n}=12)$ type 2 Diabetes mellitus, $14 \%(\mathrm{n}=12)$ hyperlipidemia and $25 \%$ $(\mathrm{n}=21)$ family history. Treatment with furosemide $85 \%$ ( $\mathrm{n}=69$ ), [ACEI/Angiotensin receptor blocker (ARB)] 92\% $(n=75)$, spironolactone $70 \%(n=57)$, beta-blockers $96 \%$ $(n=78), 14 \%(n=12)$ oral anti-diabetic $14 \%(n=12)$, statin $14 \%(n=12)$ and smokers $\% 14(n=12)$. All the patients were classified according to the NYHA (functional class I-51 patients, II-37 patients). Mean heart rate was $72 \pm 8.51$ beat per minute at the beginning of the study.

Echocardiographic characteristics were evaluated at the baseline and after six months (Table 2). The Stroke Volume increased significantly from $58.25 \pm 25.33$ to $69.07 \pm 18.43$ $\mathrm{mL} / \mathrm{m}^{2}(\mathrm{p}<0.001)$. The End-systolic volume (ESV) and end diastolic volume (EDV) decreased significantly from $128.95 \pm 53.86$ to $123.77 \pm 50.14 \mathrm{~mL} / \mathrm{m} 2(\mathrm{p}<0.001)$ and from $183.25 \pm 71.38$ to $189.96 \pm 64.17 \mathrm{~mL} / \mathrm{m}^{2} \quad(\mathrm{p}<0.001)$ respectively. We obtained quantitative measurements such as PISA, effective regurgitant orifice area (EROA),

Table 1. The baseline characteristics of 81 patients

\section{NIMR (n:81)}

\begin{tabular}{|l|l|}
\hline Age (years) & $45.85 \pm 9.86$ \\
\hline Gender \%(n) & $63(51)$ \\
\hline HT \%(n) & $44(36)$ \\
\hline DM \%(n) & $14(12)$ \\
\hline HLD \%(n) & $14(12)$ \\
\hline FH \%(n) & $25(21)$ \\
\hline Smoking \%(n) & $14(12)$ \\
\hline NYHA [I / II \% (n)] & $63(51) / 37(30)$ \\
\hline Medicine & \\
\hline Loop Diuretic \%(n) & $85(69)$ \\
\hline ACEI/ ARB \%(n) & $92(75)$ \\
\hline Spironolactone \%(n) & $70(57)$ \\
\hline Beta-blocker \%(n) & $96(78)$ \\
\hline OAD \%(n) & $14(12)$ \\
\hline Statin \%(n) & $14(12)$ \\
\hline $\begin{array}{l}\text { NIMR: Non-ischemic Mitral Regurgitation, HT: Hypertension, DM: Diabetes } \\
\text { mellitus, HLD: Hyperlipidemia, FH: Family History, NYHA: New York Heart } \\
\text { Association, ACE: Angiotensin-converting enzyme, ARB: Angiotensin receptor } \\
\text { blocker, OAD: Oral antidiabetic. }\end{array}$ \\
\hline
\end{tabular}

and $\mathrm{VC}$ from all the patients at the baseline and the one after six months. The PISA decreased significantly from $42(17-64)$ to $29(12-47) \mathrm{mm}^{2}(\mathrm{p}<0.001)$. The ROA calculated by the PISA method decreased significantly from $27(16-41)$ to $20(10-34) \mathrm{mm}^{2}(\mathrm{p}<0.001)$. The Correlation between the different changes in ERO after six months was significant $(\mathrm{r}=0.25, \mathrm{p}=0.02)$ (Figure 1). The RV calculated by the quantitative Doppler method, and VC width decreased significantly from $0.43(0.20$ $0.60)$ to $0.40(0.20-0.50) \mathrm{cm}(\mathrm{p}<0.001)$ (Table 2, Figure $2)$. The EF significantly increased from $26.25 \pm 6.08$ to $31.77 \pm 6.41 \% \quad(p<0.001)$. The 2D speckle strain was performed on the Antero-lateral papillary muscle (ALPM) and on the Postero-medial papillary muscle (PMPM) and strain values increased significantly from $9.79 \pm 2.93$ to $12.27 \pm 3.90 \% \quad(\mathrm{p}<0.001)$, and from $9.20 \pm 3.18$ to $12.25 \pm 4.41 \%(\mathrm{p}<0.001)$ respectively (Table 2, Figure $3)$. There was no significance difference at baseline and after six months for left ventricle end-diastolic diameter (LVEDD) and diastolic function parameters.

\section{Discussion}

Our study suggests that the simple 6MW is linked with the degree of the MR. In asymptomatic non-ischemic

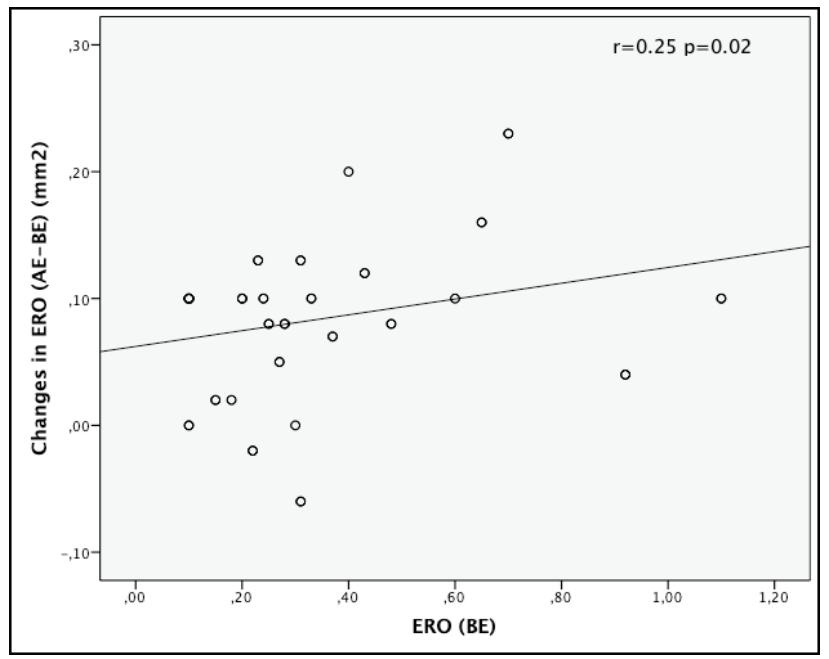

Figure 1. Correlation between changes in effective regurgitant orifice after six months of exercise and the degree of mitral regurgitation at baseline.

EROA: Effective Regurgitant Orifice Area, AE: After Exercise, BE: Before Exercise 


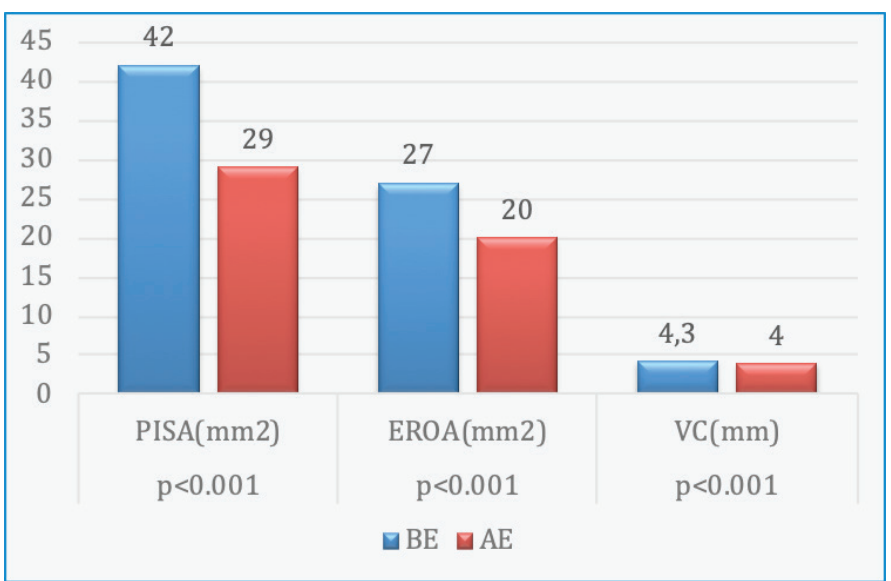

Figure 2. Quantitative Mitral Regurgitation Parameters baseline and after 6 months

PISA: Proximal Isovelocity Surface Area, EROA: Effective Regurgitant Orifice Area, VC: Vena Contracta, BE: Before Exercise, AE: After Excercise.

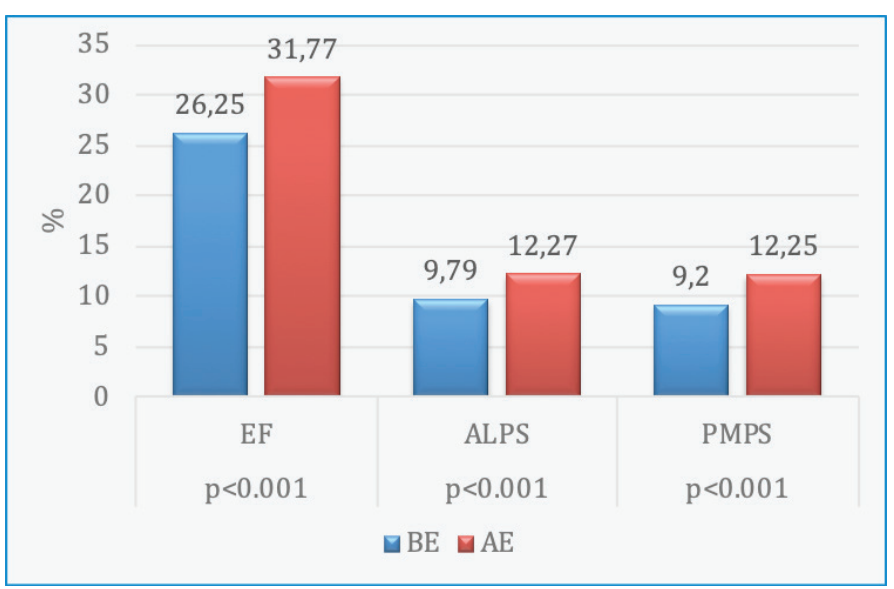

Figure 3. Strain values and Ejection Fraction changes as a percentage in MR patients

EF: Ejection Fraction, ALPS: Anterolateral papillary strain, PMPS: Posteromedial papillary strain, BE: Before Exercise, AE: After Excercise.

patients with LV dysfunction and mild or moderate mitral valve regurgitation, the $6 \mathrm{MW}$ test provides additional information to echocardiographic findings.

Functional capacity could be easily measured by the simple, inexpensive and self-paced 6MW test. As the patient determines the distance walked, it may represent the patient's everyday experience. In the HF population, the aerobic exercise has been used as an outcome measure in several clinical trials $(13,14)$. LV function has been shown to be a primary determinant of morbidity and mortality rates in MR. Leszek et al. (15) reported that 6MW test is related with the degree of the LV remodeling. Dynamic exercise studies showed increases in secondary MR in non-ischemic (16) etiology, and neither LV volume nor EF at rest or during exercise is a reliable predictor of exerciseinduced MR deterioration. In addition, progressive annular dilation during exercise may be a contributor to exercise induced MR in non-ischemic cardiomyopathy (17).

After six months, changes in ESV and LVEF were examined during the analysis of the echocardiography by using the Simpson's method. When a healthy individual exercises in a supine position, the EDV slightly increases, whereas the ESV significantly decreases. An increase in the stroke volume and the LVEF augments the cardiac output (18). There was no significant difference at baseline and after six months for LVEDD and diastolic function parameters but the stroke volume increased in the present study.

Quantitative Doppler echocardiography has proved to be a reliable method. The problem to avoid is the overestimation of the MR severity (19) and this may be due to the overestimated mitral annular area or time-velocity integral, or both. The PISA method appears to be more rapid and practical when the flow-convergence region is optimal. The flow convergence is the most recommended method (20) to evaluate MR severity. The aliasing velocity of color flow mapping is necessary for obtaining an appropriate PISA shape (21). Using quantitative Doppler echocardiography to measure the volume of regurgitation during exercise may be an alternative in patients with a semi-elliptic or asymmetric shape of the flow convergence zone. Lebrun and all the reported feasibility of measuring $\mathrm{RV}$ with the PISA method was better during exercise than at rest (22). Similarly, in this study we've reported that the PISA values became better after six months of 6MW test. We know that the PM asynchrony was associated with greater mitral RV and increased severity of MR. We've reported that the 2D speckle strain of APM and PMPM values increased significantly. 
Table 2. Echocardiographic characteristics of the patients; baseline and after six months

\begin{tabular}{|c|c|c|c|}
\hline & Baseline & 6 months & $p$ value \\
\hline EF (\%) & $26.25 \pm 6.08$ & $31.77 \pm 6.41$ & $<0.001$ \\
\hline $\mathrm{SV}\left(\mathrm{ml} / \mathrm{m}^{2}\right)$ & $58.25 \pm 25.33$ & $69.07 \pm 18.43$ & $<0.001$ \\
\hline $\operatorname{EDV}\left(\mathrm{ml} / \mathrm{m}^{2}\right)$ & $183.25 \pm 71.38$ & $189.96 \pm 64.17$ & $<0.001$ \\
\hline LV end-diastolic diameter (mm) & $6.37 \pm 0.77$ & $6.32 \pm 0.75$ & 0.103 \\
\hline LA diameter (mm) & $4.28 \pm 0.73$ & $4.08 \pm 1.04$ & 0.04 \\
\hline Mitral E-wave velocity (cm/s) & $72.30 \pm 26.83$ & $75.48 \pm 24.11$ & 0.050 \\
\hline Mitral A-wave velocity $(\mathrm{cm} / \mathrm{s})$ & $64.17 \pm 20.19$ & $65.53 \pm 27.23$ & 0.472 \\
\hline $\mathrm{PISA}\left(\mathrm{mm}^{2}\right)$ & $42(17-64)$ & $29(12-47)$ & $<0.001$ \\
\hline PMPS (\%) & $9.20 \pm 3.18$ & $12.25 \pm 4.41$ & $<0.001$ \\
\hline E-wave deceleration time (ms) & $129.88 \pm 40.91$ & $126.88 \pm 47.65$ & 0.481 \\
\hline Mitral E' (cm/s) & $6.13 \pm 2.18$ & $6.11 \pm 2.05$ & 0.912 \\
\hline Mitral $A^{\prime}(\mathrm{cm} / \mathrm{s})$ & $5.64 \pm 2.03$ & $5.64 \pm 2.11$ & 0.991 \\
\hline
\end{tabular}

ERO is a primary determinant of survival in patients with asymptomatic organic MR (20), and according to the guidelines of American College of Cardiology/ American Heart Association (23) and European Society of Cardiology (24), surgery may be recommended in selected asymptomatic patients with severe organic MR. While ERO is an anatomical entity; the RV depends on hemodynamic parameters. Both the EROA and the RV decreased significantly after six months with the $6 \mathrm{MW}$ test in our study.

In some studies, non-ischemic MR has been assessed using 2D quantitative techniques: proximal isovelocity surface (PISA) and pulsed Doppler volumetry. Underestimation of ERO in MR, low reproducibility and indirect measurement of ERO $(6,8)$ was known with several limitations for these studies. The PISA method significantly underestimated ERO due to the geometric and flow expectations in patients with functional MR. The irregular shape of ERO is relatively common in functional MR (25). Asymmetric ERO leads to the underestimation of functional MR severity by usually using the 2D methods. By dividing the RV with the MR VTI area measured with $\mathrm{CW}$ Doppler at the four-chamber view, the value of the ERO was obtained.

The presence of relatively large $\mathrm{VC}$ area (VCA) at rest is associated with adverse clinical outcome in patients with mild-moderate functional MR at rest. The assessment of VCA at rest and its increasement during exercise are independent predictors of clinical outcome. The changes in the VC width correlated well with the changes in RV. The Color flow mapping of the $\mathrm{VC}$ of the MR jet is considered being relatively quick and easy (11). Several studies have showed high feasibility and reproducibility for VCA assessment at rest (26). VCA at rest has high sensitivity while its exercise-induced increase has high specificity for identifying patients with the adverse clinical outcome 
(27). Also, the assessments of VCA seems to be highly sensitive to track exercise-induced changes in functional MR, while the PISA method underestimated EROA both at rest and during exercise. In our study we reported the VCA decreased significantly at the end of the six months with the $6 \mathrm{MW}$ test.

\section{Limitations}

Our study was done in a single medical center and therefore it's not free of selection bias. By design, patients who had not been managing exercise or patients who had symptoms at rest were not included in our study, and the results could not be generalized. Also, exercise capacity can be influenced by numerous comorbidities, including higher body mass index, not just MR. Finally, studies with a higher number of patients and novel echocardiographic modalities will be helpful to describe the appropriate assessment for functional MR.

\section{Conclusions}

Non-ischemic heart failure is often accompanied by a dynamic MR and sometimes severe MR. Six months of 6MW exercise showed a diminution in secondary MR which appears to be related to the changes in the global LV function. We have conclude that a simple, easily reproducible $6 \mathrm{MW}$ exercise might supplement the assessments of the grade of MR in secondary MR patients.

Disclosure and conflicts of interest: The authors declare no conflict of interest.

Financial support: This research did not receive any specific grant from funding agencies in the public, commercial, or not-for-profit sectors.

\section{References}

1. Iung B, Baron G, Butchart EG, et al. A prospective survey of patients with valvular heart disease in Europe: The Euro Heart Survey on Valvular Heart Disease. Eur Heart J 2003;24:1231-43.

2. Stevenson LW, Brunken RC, Belil D, et al. Afterload reduction with vasodilators and diuretics decreases mitral regurgitation during upright exercise in advanced heart failure. J Am Coll Cardiol 1990;15:174-80.

3. Grigioni F, Enriquez-Sarano M, Zehr KJ, Bailey KR, Tajik AJ. Ischemic mitral regurgitation: Long-term outcome and prognostic implications with quantitative Doppler assessment. Circulation 2001;103:1759-64.
4. Houghton AR, Harrison M, Cowley AJ, Hampton JR. Assessing exercise capacity, quality of life and haemodynamics in heart failure: do the tests tell us the same thing? Eur J Heart Fail 2002;4:289-95.

5. Quiñones MA, Otto CM, Stoddard M, Waggoner A, Zoghbi WA; Doppler Quantification Task Force of the Nomenclature and Standards Committee of the American Society of Echocardiography. Recommendations for quantification of Doppler echocardiography: a report from the Doppler Quantification Task Force of the Nomenclature and Standards Committee of the American Society of Echocardiography. J Am Soc Echocardiogr 2002;15:167-84

6. Lancellotti P, Tribouilloy C, Hagendorff A, et al. Recommendations for the echocardiographic assessment of native valvular regurgitation: an executive summary from the European Association of Cardiovascular Imaging. Eur Hear J Cardiovasc Imaging 2013;14:611-44.

7. Schiller NB, Shah PM, Crawford M, et al. Recommendations for quantitation of the left ventricle by two-dimensional echocardiography. American Society of Echocardiography Committee on Standards, Subcommittee on Quantitation of Two-Dimensional Echocardiograms. J Am Soc Echocardiogr 1989;2:358-67.

8. Zoghbi WA, Enriquez-Sarano M, Foster E, et al. Recommendations for evaluation of the severity of native valvular regurgitation with twodimensional and Doppler echocardiography. J Am Soc Echocardiogr 2003;16:777-802.

9. Enriquez-Sarano M, Miller FA JR, Hayes SN, Bailey KR, Tajik AJ, Seward JB. Effective mitral regurgitant orifice area: clinical use and pitfalls of the proximal isovelocity surface area method. J Am Coll Cardiol 1995;25:703-9.

10. Enriquez-Sarano M, Sinak LJ, Tajik AJ, Bailey KR, Seward JB. Changes in effective regurgitant orifice throughout systole in patients with mitral valve prolapse: a clinical study using the proximal isovelocity surface area method. Circulation 1995;92:2951-8.

11. Hall SA, Brickner ME, Willett DL, Irani WN, Afridi I, Grayburn PA. Assessment of mitral regurgitation severity by Doppler color flow mapping of the vena contracta. Circulation 1997;95:636-42.

12. Lang RM, Bierig M, Devereux RB, et al. Recommendations for chamber quantification: a report from the American Society of Echocardiography's Guidelines and Standards Committee and the Chamber Quantification Writing Group, developed in conjunction with the European Association of Echocardiography, a branch of the European Society of Cardiology. J Am Soc Echocardiogr 2005; 18:1440-63.

13. Wisloff U, Stoylen A, Loennechen JP, et al. Superior Cardiovascular Effect of Aerobic Interval Training Versus Moderate Continuous Training in Heart Failure Patients: A Randomized Study. Circulation 2007;115:3086-94.

14. Gielen S, Laughlin MH, O'Conner C, Duncker DJ. Exercise training in patients with heart disease: review of beneficial effects and clinical recommendations. Prog Cardiovasc Dis 2015;57:347-55.

15. Leszek P, Klisiewicz A, Janas J, et al. Usefulness of 6-minute walk test, plasma neurohumoral and cytokine activation in the assessment of symptomatic patients with left ventricle dysfunction caused by chronic severe mitral valve regurgitation. Acta Cardiol 2010;65:43-51.

16. Lapu-Bula R, Robert A, Van Craeynest D, et al. Contribution of exerciseinduced mitral regurgitation to exercise stroke volume and exercise capacity in patients with left ventricular systolic dysfunction. Circulation 2002;106:1342-8. 
17. Ennezat PV, Maréchaux S, Le Tourneau T, et al. Myocardial asynchronism is a determinant of changes in functional mitral regurgitation severity during dynamic exercise in patients with chronic heart failure due to severe left ventricular systolic dysfunction. Eur Heart J 2006;27:679-83.

18. Bonow RO, Mann DL, Zipes DP, Libby P. Braunwald's Heart Disease E-Book: A Textbook of Cardiovascular Medicine. Elsevier Health Sciences; 2011.

19. Enriquez-Sarano M, Bailey KR, Seward JB, Tajik AJ, Krohn MJ, Mays JM. Quantitative Doppler assessment of valvular regurgitation. Circulation 1993;87:841-8

20. Enriquez-Sarano M, Avierinos JF, Messika-Zeitoun D, et al. Quantitative determinants of the outcome of asymptomatic mitral regurgitation. N Engl J Med 2005;352:875-83.

21. Deng YB, Shiota T, Shandas R, Zhang J, Sahn DJ. Determination of the most appropriate velocity threshold for applying hemispheric flow convergence equations to calculate flow rate: selected according to the transorifice pressure gradient. Digital computer analysis of the Doppler color flow convergence region. Circulation 1993;88:1699-708.

22. Lebrun F, Lancellotti P, Pierard LA.ege LP-J of the A, 2001 undefined. Quantitation of functional mitral regurgitation during bicycle exercise in patients with heart failure. J Am Coll Cardiol 2001;38:1685-92.

23. American College of Cardiology; American Heart Association Task Force on Practice Guidelines (Writing Committee to revise the 1998 guidelines for the management of patients with valvular heart disease); Society of Cardiovascular Anesthesiologists, Bonow RO, Carabello BA, Chatterjee K, et al. ACC/AHA 2006 guidelines for the management of patients with valvular heart disease: a report of the American College of Cardiology/American Heart Association Task Force on Practice Guidelines (writing Committee to Revise the 1998 guidelines for the management of patients with valvular heart disease) developed in collaboration with the Society of Cardiovascular Anesthesiologists endorsed by the Society for Cardiovascular Angiography and Interventions and the Society of Thoracic Surgeons. J Am Coll Cardiol 2006;48:1-148.

24. Vahanian A, Baumgartner H, Bax J, et al. Guidelines on the management of valvular heart disease: The Task Force on the Management of Valvular Heart Disease of the European Society of Cardiology. Eur Heart J 2007;28:23068 .

25. Grayburn PA, Weissman NJ, Zamorano JL. Quantitation of mitral regurgitation. Circulation 2012;126:2005-17.

26. Marsan NA, Westenberg JJ, Ypenburg C, et al. Quantification of functional mitral regurgitation by real-time $3 \mathrm{D}$ echocardiography: comparison with $3 \mathrm{D}$ velocity-encoded cardiac magnetic resonance. JACC Cardiovasc Imaging 2009;2:1245-52.

27. Vecera J, Bartunek J, Vanderheyden M, Kotrc M, Kockova R, Penicka M. Three-dimensional echocardiography-derived vena contracta area at rest and its increase during exercise predicts clinical outcome in mild-moderate functional mitral regurgitation. Circ J 2014;78:2741-9. 